
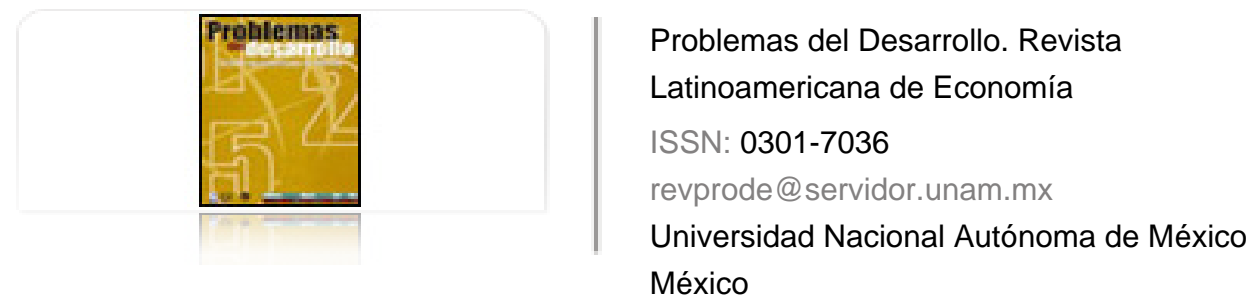

Rodríguez Padilla, Víctor

Contratos de servicios múltiples en Pemex: eficacia, eficiencia y rentabilidad Problemas del Desarrollo. Revista Latinoamericana de Economía, vol. 41, núm. 163, octubrediciembre, 2010, pp. 119-140

Universidad Nacional Autónoma de México

Distrito Federal, México

Disponible en: http://www.redalyc.org/articulo.oa?id=11819762007

- Cómo citar el artículo

Número completo

- Más información del artículo

Página de la revista en redalyc.org

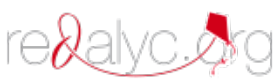

Sistema de Información Científica

Red de Revistas Científicas de América Latina, el Caribe, España y Portugal Proyecto académico sin fines de lucro, desarrollado bajo la iniciativa de acceso abierto 
Revista Problemas del Desarrollo, 163 (41), octubre-diciembre 2010

\title{
Contratos de servicios múltiples en Pemex: EFICACIA, EFICIENCIA Y RENTABILIDAD
}

\author{
Víctor Rodríguez Padilla*
}

Fecha de recepción: 22 de junio de 2010. Fecha de aceptación: 25 de septiembre de 2010.

\section{RESUMEN}

Este artículo analiza el desempeño de los Contratos de Servicios Múltiples (CSM) en términos de eficacia, eficiencia y rentabilidad para Pemex Exploración y Producción. Los resultados indican fuertes contrastes. Han conseguido atraer capacidades técnicas y financieras del sector privado, sin embargo, el volumen incremental de gas entregado por los contratistas ha quedado muy por debajo de las metas anuales, el costo de producción supera el precio del gas importado y, en seis de nueve contratos, el organismo público pierde dinero después de impuestos. Algunos objetivos secundarios se han cumplido plenamente, pero otros de manera parcial o escasa.

\section{PALABRAS CLAVE}

Petróleo, gas natural, Contratos de Servicios Múltiples, Pemex, inversión.

Profesor del Departamento de Sistemas Energéticos, División de Ingeniería Eléctrica, Posgrado de la Facultad de Ingeniería, UNAM, Correo electrónico energia123@hotmail.com 
Víctor Rodríguez Padilla

\section{Summary}

This article analyzes the performance of the MSCs in terms of efficacy, efficiency and profitability for Pemex Exploration and Production. The results indicate sharp contrasts. They have been able to attract technical and financial capacities from the private sector, but the incremental volume of gas delivered by the contractors has stayed well below the annual targets, the cost of production has surpassed the price of imported gas and, in six of the new contracts, the State corporation made after-tax losses. Some secondary goals have been fully accomplished, while others have been partly or scarcely achieved. Key words: oil, natural gas, Multiple Service Contracts, Pemex, investment.

\section{Résumé}

Cet article analyse les performances des CSM en matière d'efficacité, d'efficience et de rentabilité pour le secteur exploration et production de Pemex. Les résultats indiquent de forts contrastes. Ils sont parvenus à attirer des ressources techniques et financières du secteur privé, cependant l'accroissement du volume de gaz livré par les sous-traitants est resté très en-dessous des objectifs annuels, le coût de production dépasse le prix du gaz importé et, pour six des neufs contrats passés, l'organisme public perd de l'argent suite à des impôts. Certains objectifs secondaires ont été totalement atteints, mais d'autres l'ont été partiellement ou dans une faible mesure.

Mots clés : pétrole, gaz naturel, contrats de services multiples, Pemex, investissement.

\section{Resumo}

Este artigo analisa o desempenho dos Contratos de Serviços Múltiplos (CSM), em termos de eficácia, eficiência e rentabilidade para a Pemex Exploração e Produção. Os resultados indicam fortes contrastes. Conseguiram atrair recursos técnicos e financeiros do setor privado, no entanto, o volume incremental de gás entregue pelos contratistas ficou muito aquém das metas anuais, o custo de produção foi superior ao preço do gás importado, e em seis dos nove contratos, o público perdeu dinheiro depois de impostos. Alguns objetivos secundários foram integralmente cumpridos, mas outros, foram cobertos de modo parcial ou limitado.

Palavras-chave: petróleo, gás natural, Contratos de Serviços Múltiplos, Pemex, investimento.

\section{墨西哥国家石油公司的多服务合同：效益，效率和盈利能力 综述 \\ 本文分析了 CSM对国家石油公司勘探和生产部门，在效果，效率和盈利能 力方面的作用。结果表明强烈的反差。成功地吸引了私营部门的技术和财 \\ 政, 然而, 由承办商提供的天然气远远低于年度目标, 生产成本超过了 进口天然气的价格，并在9项合同中的6项合同，国营企业的税后损失很明 显。一些次要的（附属）目标已经全面实现，其他目标只实现一部分。 关键词：石油，天然气，多服务合同，墨西哥国家石油公司和投资}




\section{INTRODUCCIÓN}

Los Contratos de Servicios Múltiples (CSM) son una modalidad de contratación utilizada por Pemex Exploración y Producción (PEP) desde 2004. Su finalidad es incrementar las reservas y la producción de gas natural no asociado. No son contratos de servicios puros. ${ }^{1}$ Forman parte de la familia de contratos de servicios operativos (operating services contracts) aparecidos hacia finales de la década de los años ochenta en países con fuertes restricciones a la inversión privada, pero con gobiernos que por razones diversas decidieron aprovechar el capital y experiencia de las compañías petroleras internacionales. La disminución del nacionalismo, el avance del pragmatismo y el regreso de las compañías a los grandes países productores, fueron considerados en su momento elementos de un nuevo orden petrolero internacional basado en la cooperación más que en la confrontación (Bourgeois y Rodríguez-Padilla, 1992). Esa familia de contratos novedosos ha sido utilizada bajo diversos matices en Argelia, Kuwait, Irán, Irak y Venezuela (Rodríguez, 2004).

El objetivo de este artículo es evaluar el desempeño económico de los CSM en términos de eficacia, eficiencia y rentabilidad. Dicha evaluación no pretende ser exhaustiva, se limita a ciertos aspectos puntuales que pueden ser tratados con la información más completa y veraz disponible, a saber, aquélla suministrada directamente por Pemex a los órganos de fiscalización del Congreso de la Unión en respuesta a preguntas específicas sobre los objetivos de dichos contratos y sus resultados concretos entre 2004 y 2007. La dimensión jurídica, que ha dado origen a un álgido debate nacional y demandas en tribunales civiles y administrativos, no es tratada en esta investigación. El documento se organiza en cuatro partes: en la primera se analiza el origen, la justificación y los beneficios esperados de los CSM, así como sus características esenciales y el seguimiento y evaluación por parte de PEP. En la segunda parte se analiza la eficacia operativa, la eficiencia económica y la rentabilidad. En la tercera se analizan los compromisos en materia de entrenamiento, apoyo a la comunidad y contenido nacional. En la cuarta y última parte se contrasta el desempeńo de los CSM con respecto a los objetivos que se planteó PEP inicialmente.

1 Los contratos de servicio son utilizados por compañías petroleras privadas o públicas para adquirir servicios de proveedores especializados en geociencias, ingeniería petrolera y otras materias. No son acuerdos o convenios de explotación. A una compañía de servicios no se le asigna un bloque donde tendrá derechos exclusivos. El contratista no adquiere ni la propiedad de los hidrocarburos in situ, ni derechos de extracción ni derechos sobre la producción. El contratista no asume ni el riesgo geológico ni el de mercado. El pago no está atado a las reservas descubiertas, la producción o las ventas. El contrato dura el tiempo que exige la realización de la obra o la prestación del servicio, normalmente no más de tres ańos. La exclusividad espacial, temporal o profesional a favor del contratista es nula. 
Víctor Rodríguez Padilla

\section{ORIGEN Y CARACTERÍSTICAS DE LOS CSM}

\subsection{Origen, justificación y beneficios esperados}

A finales de 2001 Pemex solicitó ayuda de compañías petroleras internacionales (CPI) para incrementar las reservas y la producción de gas natural y evitar su creciente importación. La cuenca de Burgos, la principal cuenca gasífera del país, productora de gas no asociado, sería la primera en abrirse a la inversión privada mediante la licitación de ocho áreas. Las compañías participarían a través de una modalidad contractual inédita denominada CSM, de la cual ya se tenía un primer boceto que PEP afinaría con retroalimentación de las compañías interesadas. La meta era alcanzar una plataforma de producción de 1,000 millones de pies cúbicos diarios (MMpcd) y mantenerla durante seis años a partir de 2007.

A mediados de junio de 2002 se realizó una segunda conferencia internacional en la que Pemex dio a conocer el contrato genérico y reiteró su intención de aumentar la producción de gas no asociado para disminuir el déficit comercial de gas natural en el corto plazo y eliminarlo en el mediano plazo. También planteó la intención de exportar para contribuir a la seguridad energética de América del Norte (Muñoz Leos, 2002). En agosto y diciembre se publicaron nuevas versiones del contrato que tomaron en cuenta recomendaciones para disminuir el riesgo, elevar la rentabilidad y hacer más atractiva la inversión.

Durante la primera ronda (julio de 2003) se licitaron siete bloques, todos ellos con reservas probadas y producción, utilizando la quinta versión del contrato genérico. PEP asignó cinco bloques (Reynosa Monterrey, Misión, Cuervito, Fronterizo y Olmos) y dos quedaron desiertos (Ricos y Corindón-Pandura). Durante la segunda ronda (20042005) se licitaron los bloques Pandura-Anáhuac, Pirineo y Monclova. Este último se adjudicó a un consorcio mexicano-estadounidense, ${ }^{2}$ pero no se pudo firmar por causas ajenas al organismo público.

A mediados de 2005 Pemex suspendió el programa de licitación, para atender la recomendación de la Auditoría Superior de la Federación que había detectado imprecisiones jurídicas. A finales de ese año PEP consideró conveniente utilizar el término legal de Contratos de Obra Pública Financiada sobre la Base de Precios Unitarios (COPF), en lugar de CSM. En 2007 la administración de Felipe Calderón realizó una tercera ronda compuesta de tres bloques, de los cuales uno quedó desierto (Euro) y dos se asignaron (Nejo y, por segunda vez, Monclova). La escasa competencia ha caracterizado a los procesos de licitación, en siete ocasiones Pemex aceptó la oferta del único concursante participante; para Fronterizo se presentaron dos propuestas pero una fue desechada por incumplimiento de las bases de licitación; ${ }^{3}$ sólo en un caso (Pandura-Anáhuac) Pemex

2 Hullera Mexicana (Mex.), Energy Milenium (Mex.), Andrews Technologies de México (Mex.), Yuma Exploration and Production (USA), Aries Operating (USA).

3 La propuesta técnica presentada por Amistad Energy Corporation (Texas) y las empresas chinas Daquing Oilfield Limited-Downhole Service Sub-Company y Tiainjin Dagang Shengkang Petroleum Technology Development, Co. Ltd. fue desechada por no presentar la documentación completa requerida en las bases de licitación 
pudo seleccionar entre dos ofertas económicas. El cuadro 1 presenta las características generales de los nueve CSM vigentes hasta finales de 2007.

Cuadro 1. Características de los CSM firmados y vigentes en 2007

\begin{tabular}{|c|c|c|c|c|c|}
\hline Nombre de los bloques & Licitantes & echa de adjudicación & Consorcio ganador & Área $\left(\mathrm{km}^{2}\right)$ & Número de Contrato \\
\hline \multicolumn{6}{|c|}{ Primera Ronda } \\
\hline Reynosa Monterrey & 1 & $14 / 11 / 2003$ & Repsol Exploración México(Esp) & 3538 & 414103990 \\
\hline Misión & 1 & & $\begin{array}{l}\text { Servicios Múltiples de Burgos (Tecpetrol } \\
\text { (Argentina), Industrial Perforadora de } \\
\text { Campeche, Techint (Arg)) }\end{array}$ & 1972 & 414103997 \\
\hline Cuervito & 1 & $21 / 11 / 2003$ & $\begin{array}{l}\text { PTD Servicios Múltiples (Petrobras (Bra), } \\
\text { Diavaz (Mex), Teikoku (Jap)) }\end{array}$ & 231 & 414103994 \\
\hline Fronterizo & 2 & $08 / 12 / 2003$ & $\begin{array}{l}\text { PTD Servicios Múltiples (Petrobras (Bra), } \\
\text { Diavaz (Mex), Teikoku (Jap)) }\end{array}$ & 231 & 414113808 \\
\hline Olmos & 1 & $08 / 02 / 2004$ & Lewis Energy (USA) & 358 & 414104806 \\
\hline \multicolumn{6}{|c|}{ Segunda Ronda } \\
\hline Pandura-Anáhuac & 2 & $09 / 11 / 2004$ & $\begin{array}{l}\text { Industrial Perforadora de Campeche (Mex), } \\
\text { Desarrollo y Servicios Petroleros (Mex) }\end{array}$ & 1580 & 414104976 \\
\hline Pirineo & 1 & $24 / 02 / 2005$ & $\begin{array}{l}\text { Monclova Pirineos Gas (Constructora } \\
\text { Industrial Monclova (Mex), Materiales la } \\
\text { Gloria (Mex), Alianz Petroleum (Mex), } \\
\text { Steel Serv (Mex), Suelopetrol y NCT (Ven), } \\
\text { Estudios y Proyectos (Ven), Petrotesting } \\
\text { Colombia (Col)) } \\
\quad \text { Tercera Ronda }\end{array}$ & 3840 & 414105826 \\
\hline Monclova & 1 & $22 / 03 / 2007$ & $\begin{array}{l}\text { GPA Energy (Construcciones Mecánicas } \\
\text { Monclova (Mex), Administradora de } \\
\text { Proyectos de Campos (Mex) y Production } \\
\text { Testing Services (Col)) }\end{array}$ & 3358 & 424507809 \\
\hline Nejo & 1 & $15 / 03 / 2007$ & $\begin{array}{l}\text { Monclova Pirineos Gas (Mex) e } \\
\text { Iberoamericana de Hidrocarburos (Esp) }\end{array}$ & 1165 & 414107806 \\
\hline
\end{tabular}

Notas. El contrato se extiende por 20 años, pero se reduce a 15 para Cuervito, Fronterizo y Olmos.

Fuente: elaboración propia con cifras de Pemex.

Pemex justificó la estrategia de acelerar el aprovechamiento de los campos de gas no asociados mediante CSM con base en cuatro premisas (Ramírez Corso, 2001). Primero, la urgencia de expandir la producción de gas para hacer frente al rápido crecimiento de la demanda, pues de no actuar pronto las importaciones tendrían un alto costo para el país por ser más caro importar que producir; en efecto, la demanda pasaría de 4,500 a 6,700 MMpcd entre 2002 y 2006 y las importaciones alcanzarían alrededor de 2,000 MMpcd. Segundo, la escasez de capital para aumentar la producción y la total imposibilidad para obtenerlo a corto y mediano plazo debido a la astringencia del presupuesto público, el pesado régimen fiscal y los techos de endeudamiento ligados a las políticas macroeconómicas. 
Víctor Rodríguez Padilla

Tercero, la necesidad de tecnología sofisticada y experiencia externa para desarrollar de manera rentable la cuenca de Burgos, caracterizada geológicamente por pequeńos yacimientos lenticulares de baja permeabilidad que exigen la perforación continua de pozos para mantener el nivel deseado de producción. Cuarto, la conveniencia de enviar una señal a los mercados sobre la continuidad de los cambios estructurales en el país.

El nuevo esquema contractual -distinto y complementario a los utilizados hasta entonces- fue diseńado para que el sector privado aportara capacidades operativas, tecnológicas y financieras adicionales a las de PEP. La idea central consistió en contratar un amplio paquete de servicios de desarrollo y explotación de yacimientos realizados por un solo contratista. El contrato contendría términos y condiciones para: $i$ ) alentar un desempeño más consistente con el interés de PEP (aumentar la producción); ii) permitir el repago del capital invertido a partir de los ingresos obtenidos por la venta de la producción; iii) imprimir celeridad al desarrollo de los yacimientos y, iv) reducir el costo de los servicios mediante un proceso de licitación altamente competido. El nuevo esquema también le permitiría a PEP: i) disminuir los costos administrativos al compactarse cientos de contratos en unos cuantos; ii) mejorar las condiciones de financiamiento; iii) conseguir un costo de suministro por debajo del precio de importación; iv) disminuir los costos de producción al incrementarse la eficiencia de las operaciones y, $v$ ) reducir el impacto de la deuda pública. A lo anterior se agregaría un flujo de efectivo positivo para el Estado, acceso a tecnologías de punta, explotación optimizada de yacimientos, creación de empleos y mayor demanda de insumos nacionales.

\subsection{Características esenciales}

Mediante un Contrato de Servicios Múltiples, PEP, encarga a una compañía petrolera, nacional o extranjera, la búsqueda y extracción de gas natural no asociado en un área donde el contratista es el único facultado para realizar esas actividades. Gas y condensados son entregados a PEP para su comercialización. El contrato comprende el ciclo completo de las actividades de exploración y producción y tiene una duración de entre 15 y 20 ańos. El contratista se desempeńa como operador; aporta capital, tecnología, y personal; goza de libertad para subcontratar todo con excepción de la administración del proyecto. Además, debe realizar una serie de inversiones y trabajos obligatorios. Recibe incentivos para que explore y maximice la producción. El reembolso de inversiones, costos y gastos queda condicionado a que el proyecto genere ingresos pues la única fuente de ingresos es la venta de la producción. El monto que se paga mensualmente, sujeto a reglas y límites precisos, depende del volumen de trabajos realizados y de un catálogo de precios unitarios. El pago de los derechos de extracción de hidrocarburos queda a cargo de PEP; ${ }^{4}$ en cambio, el contratista sólo está sujeto al impuesto sobre la renta.

4 Ley Federal de Derechos, capítulo XII, Hidrocarburos. 
El contrato establece que no otorga ni constituye derecho alguno para explorar, explotar o producir hidrocarburos. La relación de jerarquía se establece al reconocer las partes que las actividades de exploración, explotación, elaboración y ventas de primera mano de gas constituyen actividades reservadas a la nación mexicana, de ahí que el contratista ejecutará los trabajos para beneficio de PEP, el cual ejercerá el control y supervisión permanente de las obras y tomará las decisiones fundamentales según lo requiera el contrato. Los últimos tres contratos precisan que el contratista actúa por cuenta y orden de PEP. El texto contractual señala que no otorga derecho alguno a favor del contratista sobre los yacimientos, las reservas e hidrocarburos del área de trabajo, que son propiedad exclusiva de PEP, al igual que los pozos y la infraestructura. El contrato excluye explícitamente la posibilidad de que el contratista reciba porcentajes en los hidrocarburos producidos, participe o reciba beneficios derivados de los resultados de la explotación y establece que los pagos serán en efectivo.

\subsection{Seguimiento y evaluación}

PEP no cuenta con un informe específico sobre el desempeño operativo de los CSM. Sus resultados se incluyen en la evaluación de la cuenca de Burgos junto con los proyectos Burgos Tradicional y Burgos Integral. A partir de 2006 aparece la primera información desagregada aunque de manera incompleta. De las cifras de 2007 se concluye un mayor seguimiento (cuadro 2), pero los indicadores utilizados resultan insuficientes, asistemáticos y no siempre cuantificados. ${ }^{5}$

PEP proporciona a los contratistas la Célula General del Contrato de Obra Pública donde se resume el Programa Anual de Trabajo (PAT) que debe autorizar el organismo. El formato no es homogéneo, cambia de contrato a contrato y según el año. Los mayores contrastes ocurren para Misión, Cuervito, Fronterizo y Nejo. Indicadores importantes no han sido reportados por los contratistas. ${ }^{6}$

En 2007 el Sistema Integral de Costos de PEP estaba en proceso de reestructuración y contaba con información incompleta. En consecuencia, no disponía de información

5 Por ejemplo, PEP no cuantifica las pérdidas técnicas y no técnicas entre la boca del pozo y los puntos de transferencia; tampoco conoce el autoconsumo que hacen los contratistas. La cuantificación, valuación y destino de los condensados presenta lagunas, zonas grises e incongruencias. El tratamiento de los hidrocarburos en el contrato es confuso. Por otra parte, la contabilidad presenta grandes fallas, por ejemplo, el Sistema para Integrar Programas Operativos registra un agudo faltante en la producción, pues sólo se incluye el flujo del bloque Nejo; las cifras de la producción son incongruentes y presentan diferencias de hasta mil por ciento. Por otra parte, las estadísticas no permiten conocer ni la calidad del gas entregado por los contratistas en los puntos de transferencia, ni la estructura de los ingresos por la venta de los hidrocarburos que entregan los contratistas.

6 Es el caso de los indicadores siguientes: valor presente neto (VPN), valor presente de la inversión (VPI), relación VPN/VPI, tasa interna de retorno, periodo de recuperación y relación costo beneficio. Las obligaciones financieras asociadas a los programas de capacitación al personal y los de apoyo a la comunidad no son incluidas sistemáticamente en los programas de trabajo. 
Víctor Rodríguez Padilla

Cuadro 2. PEP, evaluación el desempeño del proyecto Burgos

\begin{tabular}{|c|c|c|c|c|c|c|c|c|}
\hline & & & & & & & & CSM \\
\hline BURGOS TRADICIONAL & 2001 & 2002 & 2003 & 2004 & 2005 & 2006 & 2007 & 2007 \\
\hline Sísmica 2D (km) & 2906 & 2638 & 1143 & 4117 & 2833 & 733 & 0 & 0 \\
\hline Sísmica 3D (km) & 1594 & 499 & 1197 & 7441 & 926 & 526 & 0 & 750 \\
\hline Localizaciones aprobadas & 62 & 81 & 61 & 60 & 43 & nd & nd & Nd \\
\hline Reservas $3 p$ incorporadas (MMbpce) & 111 & 86 & 194 & 138 & 398 & 340 & 259 & 22 \\
\hline Pozos de exploración perforados & 30 & 26 & 38 & 41 & 22 & 24 & 16 & 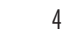 \\
\hline Pozos de exploración terminados & 36 & 27 & 28 & 40 & 28 & 30 & 13 & 4 \\
\hline Pozos de desarrollo perforados & 371 & 329 & 364 & 351 & 366 & 349 & 196 & 45 \\
\hline Pozos de desarrollo terminados & 362 & 343 & 344 & 359 & 345 & 198 & 322 & 47 \\
\hline Producción de gas (MMpcd) & 990 & 1007 & 1031 & 1094 & 1115 & 1193 & 1229 & 185 \\
\hline Condensados (Mbd) & 3.4 & 5.7 & 7.7 & 9.1 & 10.5 & nd & nd & nd \\
\hline Costo de extracción (US\$/Mpc) & 0.61 & 0.66 & 0.56 & 0.71 & 0.78 & nd & nd & nd \\
\hline Ingresos (MM\$) & 15185 & 1580 & 20322 & 25115 & 32309 & nd & nd & nd \\
\hline Utilidades antes de impuestos (MMS) & 10058 & 3266 & 14460 & 18271 & 21616 & nd & nd & nd \\
\hline BURGOS INTEGRAL & 2001 & 2002 & 2003 & 2004 & 2005 & 2006 & 2007 & 2007 \\
\hline Sísmica 2D (km) & 2906 & 2638 & 1143 & 4117 & 4299 & 733 & 0 & 0 \\
\hline Sísmica 3D (km) & 1594 & 499 & 1197 & 7441 & 1438 & 1473 & 1392 & 750 \\
\hline Localizaciones aprobadas & 62 & 81 & 61 & 60 & nd & nd & nd & $\mathrm{Nd}$ \\
\hline Reservas $3 p$ Incorporadas (MMbpce) & 111 & 86 & 194 & 106 & 398 & 354 & 281 & 22 \\
\hline Pozos de exploración perforados & 30 & 26 & 38 & 41 & 26 & 29 & 17 & 4 \\
\hline Pozos de exploración terminados & 36 & 27 & 28 & 40 & 28 & 35 & 25 & 4 \\
\hline Pozos de desarrollo perforados & 371 & 329 & 364 & 373 & 427 & 402 & 227 & 45 \\
\hline Pozos de desarrollo terminados & 362 & 343 & 344 & 371 & 401 & 386 & 267 & 47 \\
\hline Producción de gas (MMpcd) & 990 & 1007 & 1031 & 1095 & 1217 & 1331 & 1414 & 185 \\
\hline Condensados (Mbd) & 3.4 & 5.7 & 7.7 & 9.1 & 10.5 & nd & nd & Nd \\
\hline Costo de extracción (US\$/Mpc) & nd & nd & nd & nd & 0.8 & nd & nd & Nd \\
\hline Ingresos (MM\$) & nd & nd & nd & nd & 38612 & nd & nd & Nd \\
\hline Utilidades antes de impuestos (MM\$) & nd & nd & nd & nd & 21370 & nd & nd & Nd \\
\hline BURGOS INTEGRAL (éxito exploratorio) & 2001 & 2002 & 2003 & 2004 & 2005 & 2006 & 2007 & 2007 \\
\hline Pozos exploratorios & 36 & 27 & 28 & 40 & nd & 35 & 25 & 1 \\
\hline \% de éxito comercial & 56 & 48 & 57 & 35 & nd & 29 & 65 & 31 \\
\hline \% de éxito geológico & 64 & 74 & 68 & 65 & nd & 46 & nd & nd \\
\hline Costo de descubrimiento 3P (dls/Mpc) & 0.15 & 0.17 & 0.16 & 0.59 & nd & nd & nd & nd \\
\hline Costo de descubrimiento 2P (dls/Mpc) & 0.33 & 0.43 & 0.34 & 1.43 & nd & nd & nd & nd \\
\hline Inversiones (MM\$) & 946 & 914 & 1767 & 3630 & nd & nd & nd & nd \\
\hline
\end{tabular}

Burgos Integral coincide con Burgos Tradicional para los años 2001, 2002 y 2003. Los (SM iniciaron en 2004 pero la información comienza a reportarse en 2006. Unidades: millones de barriles de petróleo crudo equivalente (MMbpce); millones de dólares (MM\$); kilómetros (km); millones de pies cúbicos diarios (MMpcd); dólares (\$); miles de pies cúbicos (Mpc).

Fuente: elaboración propia con cifras de Pemex. 
de costos de exploración y explotación por cada campo de extracción de petróleo crudo y gas natural (ASF, 2008: 74). Por lo que toca al costo de descubrimiento no se elaboran reportes periódicos donde aparezca esa información, ya que es un indicador que no cuenta con una metodología y una periodicidad de cálculo definida y oficializada en PEP; esporádicamente se calcula cuando se requiere incluirlo en algún análisis o presentación. De igual modo, PEP no dispone de información para evaluar el beneficio bruto de cada uno de los contratos pues los indicadores se obtienen a nivel del activo integral. Cuenta con información fragmentada sobre la cantidad y composición del personal que emplean los contratistas pero desconoce el contenido nacional de los bienes y servicios que adquieren. De lo anterior se concluye un seguimiento y evaluación deficiente.

\section{EFICACIA, EFICIENCIA Y RENTABILIDAD}

\subsection{Eficacia operativa}

Para evaluar la efectividad de los CSM analizamos cuatro indicadores: la incorporación de reservas, la perforación y terminación de pozos, así como el volumen de producción entregado a PEP.

Los contratistas han desarrollando un esfuerzo exploratorio limitado; su atención se ha concentrado en el desarrollo de los yacimientos descubiertos por PEP. La excepción ha sido el bloque Olmos, donde el contratista ha perforado 3 pozos exploratorios. ${ }^{7} \mathrm{El}$ aumento de $51 \%$ en las reservas probadas para los primeros seis contratos en el periodo 2004-2008 (cuadro 3), se explica por la disminución de las reservas probables y posibles, las cuales cayeron $21.9 \%$ y $17.6 \%$ respectivamente. En total las reservas $3 \mathrm{P}$ han decrecido 1.6\%. Las diferencias entre bloques son notables. El bloque Misión, Cuervito y Fronterizo han registrado aumentos positivos en los tres tipos de reservas.

Cuadro 3. CSM, reservas probadas, probables y posibles al $1^{0}$ de enero de cada año

\begin{tabular}{lcrrrrrrr}
\hline & \multicolumn{3}{c}{2004} & \multicolumn{4}{c}{2008} \\
\cline { 2 - 8 } & Probadas & Probables & Posibles & \multicolumn{1}{c}{ Total } & Probadas & Probables & Posibles & Total \\
\hline Reynosa Monterrey & 55.0 & 110.6 & 100.0 & 265.6 & 69.1 & 35.6 & 53.3 & 158.0 \\
Misión & 63.4 & 64.7 & 77.3 & 205.4 & 114.4 & 124.6 & 81.4 & 320.4 \\
Cuervito & 36.9 & 46.6 & 15.6 & 99.0 & 45.3 & 42.2 & 41.3 & 128.8 \\
Fronterizo & 16.8 & 6.1 & 0.0 & 22.9 & 47.9 & 22.6 & 13.5 & 84.0 \\
Pandura-Anáhuac & 38.2 & 52.9 & 44.6 & 135.7 & 20.2 & 4.7 & 6.2 & 31.1 \\
Pirineo & 20.4 & 32.4 & 131.8 & 184.5 & 52.7 & 14.9 & 108.6 & 176.2 \\
Total & 230.7 & 313.3 & 369.3 & 913.1 & 349.6 & 244.6 & 304.3 & 898.5 \\
\hline
\end{tabular}

Fuente: elaboración propia con cifras de Pemex.

7 Esos pozos se denominan contractualmente de "alto riesgo". 
Víctor Rodríguez Padilla

En 2006 los CSM consiguieron incorporar reservas 3P por 7.0 Gpc por pozo, en cambio el proyecto Burgos Integral operado por PEP incorporó casi cinco veces más (34.9 Gpc por pozo). Ese contraste traduce diferencias geológicas y experiencia en la cuenca. En 2007 la brecha se redujo un poco pero seguía siendo significativa: los CSM consiguieron incorporar 9.9 Gpc de reservas $3 \mathrm{P}$ mientras que el proyecto Integral Burgos logró tres veces más $(31.5 \mathrm{Gpc})$. Ese último año el déficit entre lo planeado y lo obtenido alcanzó 78.9\% para los CSM, en cambio para Burgos Tradicional sólo llegó a 19.9\%, lo cual ya no se explica por diferencias geológicas pues los planes los define el propio contratista.

La perforación y la terminación de pozos han quedado por debajo de lo establecido por los programas anuales de trabajo. Considerando la totalidad de los bloques, en el periodo 2004-2007 se programó la perforación de 228 pozos pero sólo se realizaron 183, lo cual arroja un déficit de $19.7 \%$. Por lo que toca a la terminación de pozos, la diferencia fue de $18.3 \%$, ya que se programaron 240 terminaciones y sólo se realizaron 196. Todos los contratistas han incurrido en incumplimientos en ambos indicadores, sin embargo, los bloques Olmos, Pandura-Anáhuac y Pirineo observan los déficits más profundos y sistemáticos. El bloque Misión es el de mejor desempeño, con déficits en el año de arranque, pero superávits en años subsecuentes, particularmente importantes en 2007.

La introducción de los CSM significó una caída inmediata de $34.1 \%$ en la producción de las áreas que operan bajo ese esquema; el volumen extraído pasó de 88.4 a 58.3 MMpcd entre 2003 y 2004. En los años siguientes se revirtió la tendencia pero sin alcanzar las metas que justificaron la aprobación de la nueva modalidad contractual. Luego de tres rondas de licitaciones los CSM produjeron 189.3 MMpcd en 2007, lo cual significa $18.9 \%$ de la meta original de obtener $1000 \mathrm{MMpcd}$ a partir de ese año. ${ }^{8}$ Del gas producido en 2007, el $50.3 \%$ provino de los pozos que habían sido perforados por PEP antes de los CSM y, el restante $49.7 \%$, de los nuevos pozos perforados por los contratistas. Las metas de producción plasmadas en los programas anuales de trabajo no se han cumplido en ninguno de los años del periodo 2004-2007 (cuadro 4); los déficits van de 17.1\% a 27.3\%. En 2007 la situación mejoró pero la producción también quedó por debajo de lo planeado. Todos los bloques presentan déficits, con excepción de Misión que observa superávit en 2006 y 2007. Las brechas más importantes (entre 50 y 100\%) las registran Olmos, Pandura-Anáhuac y Pirineo. Los programas de producción de condensados tampoco se han cumplido.

\subsection{Eficiencia económica}

Para evaluar la eficiencia económica de los CSM en esta sección analizamos, primero, el cumplimiento de dos cláusulas contractuales destinadas a proteger la economía de PEP y, segundo, el costo del gas que los contratistas le entregan al organismo público.

8 La producción debía alcanzar 250 MMpcd en 2004, 500 MMpcd en 2005, 750 MMpcd en 2006 y 1000 MMpcd en 2007 y los seis ańos siguientes. 
Contratos de servicios múltiples en Pemex: eficacia, eficiencia y rentabilidad

Cuadro 4. CSM, producción de gas programada y entregada

(Millones de pies cúbicos diarios y porcentaje)

\begin{tabular}{|c|c|c|c|c|c|c|c|c|c|c|}
\hline & $\begin{array}{l}\text { Reynosa } \\
\text { Monterrey }\end{array}$ & Misión & Cuervito & Fronterizo & Olmos & $\begin{array}{l}\text { Pandura } \\
\text { Anahuac }\end{array}$ & Pirineo & Monclova & Nejo & Total \\
\hline \multicolumn{11}{|l|}{2004} \\
\hline Programa & 14 & 34.8 & 16.5 & 8.2 & na & na & na & na & na & 73.5 \\
\hline Real & 8.8 & 28.6 & 13.9 & 6.8 & na & na & na & na & na & 58.1 \\
\hline Diferencia & $-37.1 \%$ & $-17.8 \%$ & $-15.8 \%$ & $.17 .1 \%$ & na & na & na & na & na & $-21.0 \%$ \\
\hline \multicolumn{11}{|l|}{2005} \\
\hline Programa & 37.4 & 44.2 & 29 & 10.9 & na & 11.4 & 5.7 & na & na & 138.6 \\
\hline Real & 19.8 & 42.6 & 19.2 & 9.8 & na & 8.1 & 2.6 & na & na & 102.1 \\
\hline Diferencia & $-47.1 \%$ & $-3.6 \%$ & $-33.8 \%$ & $-10.1 \%$ & na & $-28.9 \%$ & $-54.4 \%$ & na & na & $-26.3 \%$ \\
\hline \multicolumn{11}{|l|}{2006} \\
\hline Programa & 61.1 & 47.2 & 18.6 & 12.3 & 11.1 & 28.2 & 10.7 & na & na & 189.2 \\
\hline Real & 37.8 & 50.1 & 17.2 & 10.5 & 0 & 16.9 & 5.1 & na & na & 137.6 \\
\hline Diferencia & $-38.1 \%$ & $6.1 \%$ & $-7.5 \%$ & $-14.6 \%$ & $-100.0 \%$ & $-40.1 \%$ & $-52.3 \%$ & na & na & $-27.3 \%$ \\
\hline \multicolumn{11}{|l|}{2007} \\
\hline Programa & 62.7 & 56.2 & 18.7 & 12.3 & 3.1 & 26.2 & 12.8 & 15.1 & 21.3 & 228.4 \\
\hline Real & 45.3 & 61.7 & 18.3 & 11.4 & 0 & 18 & 5.8 & 14.3 & 14.5 & 189.3 \\
\hline Diferencia & $-27.8 \%$ & $9.8 \%$ & $-2.1 \%$ & $-7.3 \%$ & $-100.0 \%$ & $-31.3 \%$ & $-54.7 \%$ & $-5.3 \%$ & $-31.9 \%$ & $-17.1 \%$ \\
\hline
\end{tabular}

Fuente: elaboración propia con cifras de Pemex.

Cuando se trata de obras nuevas el contrato establece que el reembolso de costos operativos y de capital está sujeto a un límite de pago mensual (LPM). En la práctica esa regla no siempre se ha cumplido. Cinco contratos han tenido pagos superiores a ese límite, especialmente en los casos de Pandura-Anáhuac y Pirineo; el exceso va de los 38 mil dólares hasta casi 2 millones de dólares y ha llegado a representar $405 \%$ con respecto a ese límite. Las reglas de cálculo tampoco se han cumplido estrictamente. Para Reynosa Monterrey, Misión, Cuervito, Fronterizo y Olmos el contrato establece un procedimiento de ajuste del LPM cuando la cantidad mínima de gas acumulado no cumple las expectativas de PEP. Dicha expectativa era recibir 1000 MMpcd en 2007, sin embargo, la forma de calcular el LPM no se modificó a pesar de que PEP sólo recibió 189.5 MMpcd.

En ocho de nueve contratos el pago al contratista ha provenido de los recursos generados por la venta de la producción. En el caso de Olmos, PEP ha pagado 15.7 millones de dólares sin que el área contractual haya generado algún ingreso, pues no ha logrado producir hidrocarburos.

Los costos promedio de producción en los CSM son cuatro veces más grandes que los costos promedios observados de la Región Norte (ASF, 2008: 72). Esa brecha podría explicarse por diferencias en estructura de costos, etapa de inversión y la riqueza geológica. 
Víctor Rodríguez Padilla

Monclova y Nejo reportaron costos muy similares a los costos regionales por estar en su primer año de operación y con un programa de inversión aún incipiente (cuadro 5).?

Cuadro 5. CSM, costos de producción

(Dólares por millar de pies cúbicos)

\begin{tabular}{lcccc}
\hline & 2004 & 2005 & 2006 & 2007 \\
\hline Reynosa Monterrey & 0.94 & 0.86 & 0.70 & 0.63 \\
Misión & 0.39 & 0.53 & 0.61 & 0.60 \\
Cuervito & 0.37 & 0.55 & 0.75 & 0.66 \\
Fronterizo & 0.66 & 0.80 & 0.89 & 0.96 \\
Olmos & 0.00 & 0.00 & 0.00 & 0.00 \\
Pandura-Anáhuac & & 0.52 & 0.54 & 0.58 \\
Pirineo & & 0.46 & 0.67 & 0.76 \\
Monclova & & & & 0.04 \\
Nejo & & & & 0.03 \\
Total & 0.50 & 0.62 & 0.67 & 0.56 \\
Región Norte & 0.10 & 0.11 & 0.09 & 0.09 \\
\hline Fuente: Sistema Integral de Costos de la Subdirección de Administración y Finanzas. & &
\end{tabular}

El costo instantáneo de producción, ${ }^{10}$ definido como el egreso anual en el que incurre por PEP entre la producción de gas que recibió del contratista, se triplicó en sólo tres años al pasar de 1.12 dólares en 2004 a 3.59 dólares en 2007 (cuadro 6). ${ }^{11}$ Ese aumento se explica por las inversiones realizadas y, en menor medida, por el proceso inflacionario que generó la subida de los precios del petróleo en el mercado internacional; ${ }^{12}$ en 2007 superó en 39\% al costo promedio de importación (2.58 dólares); en el periodo 20042007 promedió 3.11 dólares, lo cual rebasó el límite establecido para la deducción de costos permitido por el régimen fiscal petrolero (2.70 dólares).

9 Los costos muy bajos en Monclova y Nejo en 2007 se explican porque los egresos están constituidos únicamente por mantenimiento de la producción existente al inicio del contrato; se trata de costos operativos de una producción ya amortizada que PEP transfiere al contratista.

10 El costo instantáneo de producción es pertinente en el caso de la cuenca de Burgos porque el tiempo de perforación es de unas cuantas semanas y se cuenta rápidamente con producción. En otras palabras, la brecha entre inversión y producción es muy corto.

11 Los pagos incluyen erogaciones (por amortización, intereses y mantenimiento), sujetos y no sujetos al límite de pago mensual.

12 Cabe destacar que el componente inflacionario de dicho aumento fue muy reducido pues el índice de precios aplicado al catálogo de precios unitarios se situó entre 6.5 y 18.5\%. Dicho aumento correspondiente al ipp utilizado para ajustar el catalogo de precios unitarios. 
Contratos de servicios múltiples en Pemex: eficacia, eficiencia y rentabilidad

Cuadro 6. CSM, costo instantáneo de producción

(Dólares de 2007 por millar de pies cúbicos)

\begin{tabular}{|c|c|c|c|c|c|}
\hline & 2004 & 2005 & 2006 & 2007 & Promedio 2004-2007* \\
\hline & \multicolumn{5}{|c|}{ Dólares corrientes } \\
\hline Reynosa Monterrey & 2.08 & 4.01 & 4.51 & 5.11 & 4.57 \\
\hline Misión & 0.87 & 1.73 & 2.82 & 3.21 & 2.45 \\
\hline Cuervito & 0.83 & 2.23 & 3.36 & 3.66 & 2.68 \\
\hline Fronterizo & 1.48 & 2.77 & 3.63 & 4.89 & 3.49 \\
\hline \multicolumn{6}{|l|}{ Olmos } \\
\hline Pandura-Anáhuac & & 1.15 & 3.02 & 4.59 & 3.37 \\
\hline Pirineo & & 1.03 & 3.41 & 4.93 & 3.66 \\
\hline Mondlova & & & & 0.08 & 0.08 \\
\hline Nejo & & & & 0.07 & 0.07 \\
\hline Total & 1.12 & 2.30 & 3.64 & 3.59 & 3.11 \\
\hline
\end{tabular}

\subsection{Rentabilidad}

Para evaluar el atractivo económico para PEP de la ejecución de los CSM en este apartado analizamos el excedente de operación y el margen de beneficios antes y después de impuesto.

Los ingresos totales acumulados durante el periodo 2004-2007 alcanzan 1,101 millones de dólares constantes de 2007, lo cual permitió obtener un excedente de operación de 547 millones de dólares antes de impuestos que representa 50.3\% de los ingresos (cuadro 7).

El margen de beneficio para PEP antes de impuestos ha sido positivo en ocho de los nueve contratos en el periodo 2004-2007. En el caso de Olmos el organismo registró una pérdida neta de casi 14.6 millones de dólares. Cabe destacar que la dinámica de los ingresos y los egresos ha sido distinta: los primeros crecieron a un ritmo promedio anual de $51.1 \%$, en cambio, los segundos lo hicieron a más del doble (112.0\%), en consecuencia, el margen de Pemex se ha reducido. Para el conjunto de bloques los egresos absorben la mitad de los ingresos, pero en el caso de Reynosa Monterrey llegan a representar $73.2 \%$. En el extremo opuesto se encuentra Misión donde PEP obtuvo un margen de beneficio superior al $60 \% .^{13}$

13 Los casos de Nejo y Monclova deben considerarse aparte pues se adjudicaron en 2007 y los ingresos superaron por mucho los egresos. 
Víctor Rodríguez Padilla

Cuadro 7. CSM, ingresos y egresos en el periodo 2004-2007

(Dólares constantes de 2007)

\begin{tabular}{lrrrrrc}
\hline & $\begin{array}{c}\text { Ingresos totales } \\
\text { generados }\end{array}$ & \multicolumn{1}{c}{ TMCA } & \multicolumn{1}{c}{ Egresos } & TMCA & $\begin{array}{c}\text { Egresos/ } \\
\text { Ingresos }\end{array}$ & $\begin{array}{c}\text { Margen para PEP } \\
\text { 1-(Egresos/Ingreso) } \\
\text { antes de impuestos }\end{array}$ \\
\hline Reynosa Monterrey & $254,906,116$ & $75.6 \%$ & $186,672,201$ & $125.6 \%$ & $73.2 \%$ & $26.8 \%$ \\
Misión & $415,515,438$ & $32.1 \%$ & $163,890,536$ & $93.4 \%$ & $39.4 \%$ & $60.6 \%$ \\
Cuervito & $149,209,886$ & $9.9 \%$ & $67,254,161$ & $74.0 \%$ & $45.1 \%$ & $54.9 \%$ \\
Fronterizo & $88,288,737$ & $22.0 \%$ & $49,193,340$ & $71.5 \%$ & $55.7 \%$ & $44.3 \%$ \\
Olmos & 0 & & $14,585,652$ & & & \\
Pandura-Anáhuac & $99,583,392$ & $31.1 \%$ & $52,902,238$ & $187.1 \%$ & $53.1 \%$ & $46.9 \%$ \\
Pirineo & $31,887,706$ & $26.8 \%$ & $17,966,543$ & $217.4 \%$ & $56.3 \%$ & $43.7 \%$ \\
Monclova & $30,339,301$ & & 438,421 & & $1.4 \%$ & $98.6 \%$ \\
Nejo & $30,844,402$ & & 392,151 & & $1.3 \%$ & $98.7 \%$ \\
Total & $1,100,574,978$ & $51.1 \%$ & $553,295,244$ & $112.0 \%$ & $50.3 \%$ & $49.7 \%$ \\
\hline
\end{tabular}

Fuente: elaboración propia con cifras de Pemex.

Aunque el margen ex ante para PEP ha sido siempre positivo (cuadro 8), el margen ex post ha sido negativo durante los últimos años. ${ }^{14}$ La pérdida total después de impuestos fue de 13.9 millones de dólares en 2006 y de 6.6 millones de dólares en 2007. Reynosa Monterrey es el contrato con mayores pérdidas para el organismo público después de impuestos. Durante los cuatro años del periodo ha tenido pérdidas, que acumulan más 42 millones de dólares de 2007. En cambio, Misión es el contrato que representa las mayores ganancias para PEP después de impuestos (más de 40 millones de dólares de 2007) y la posibilidad de compensar las pérdidas de los otros contratos aunque no completamente. Fronterizo ha generado pérdidas desde 2005 y Cuervito en 2006 y 2007. Ese último año Pandura-Anáhuac y Pirineo representaron pérdidas, en cambio Monclova y Nejo reportaron ganancias en virtud de un reembolso de inversiones poco significativo, al ser el primer año de actividades en ambos bloques. Así, algunos contratos compensan las pérdidas en otros, en una situación similar a la de subsidios cruzados que, de cualquier manera, resultan insuficientes y derivan en pérdida neta después de impuestos para PEP.

14 La carga fiscal se determinó conforme al régimen fiscal aplicable a la fase extractiva, de acuerdo con lo establecido en la Ley de Ingresos para 2004 y 2005 (antiguo régimen fiscal de Pemex) y a la Ley Federal de Derechos para 2006 y 2007 (nuevo régimen fiscal) 2004. Antiguo Régimen Fiscal (60.8\% del ingreso bruto). 2005. Antiguo Régimen Fiscal (60.8\% del ingreso bruto). 2006. Derecho Ordinario sobre Hidrocarburos (рон). Aplica la tasa de $78.68 \%$ a la diferencia que resulte entre el valor anual del petróleo crudo y gas natural extraídos en el ańo y las deducciones permitidas: para gas natural no asociado hasta 2.70 dólares por millar de pies cúbicos Todos los demás derechos son acreditables o deducibles respecto al рон, 2007. Derecho Ordinario sobre Hidrocarburos. Aplica la tasa de $78.76 \%$ Deducciones permitidas para gas natural no asociado hasta 2.70 dólares por millar de pies cúbicos, y un monto adicional de 0.50 dólares por cada millar de pie cúbico de gas natural no asociado adicional al volumen extraído en 2006. Todos los demás derechos son acreditables o deducibles respecto al DOH. 
Contratos de servicios múltiples en Pemex: eficacia, eficiencia y rentabilidad

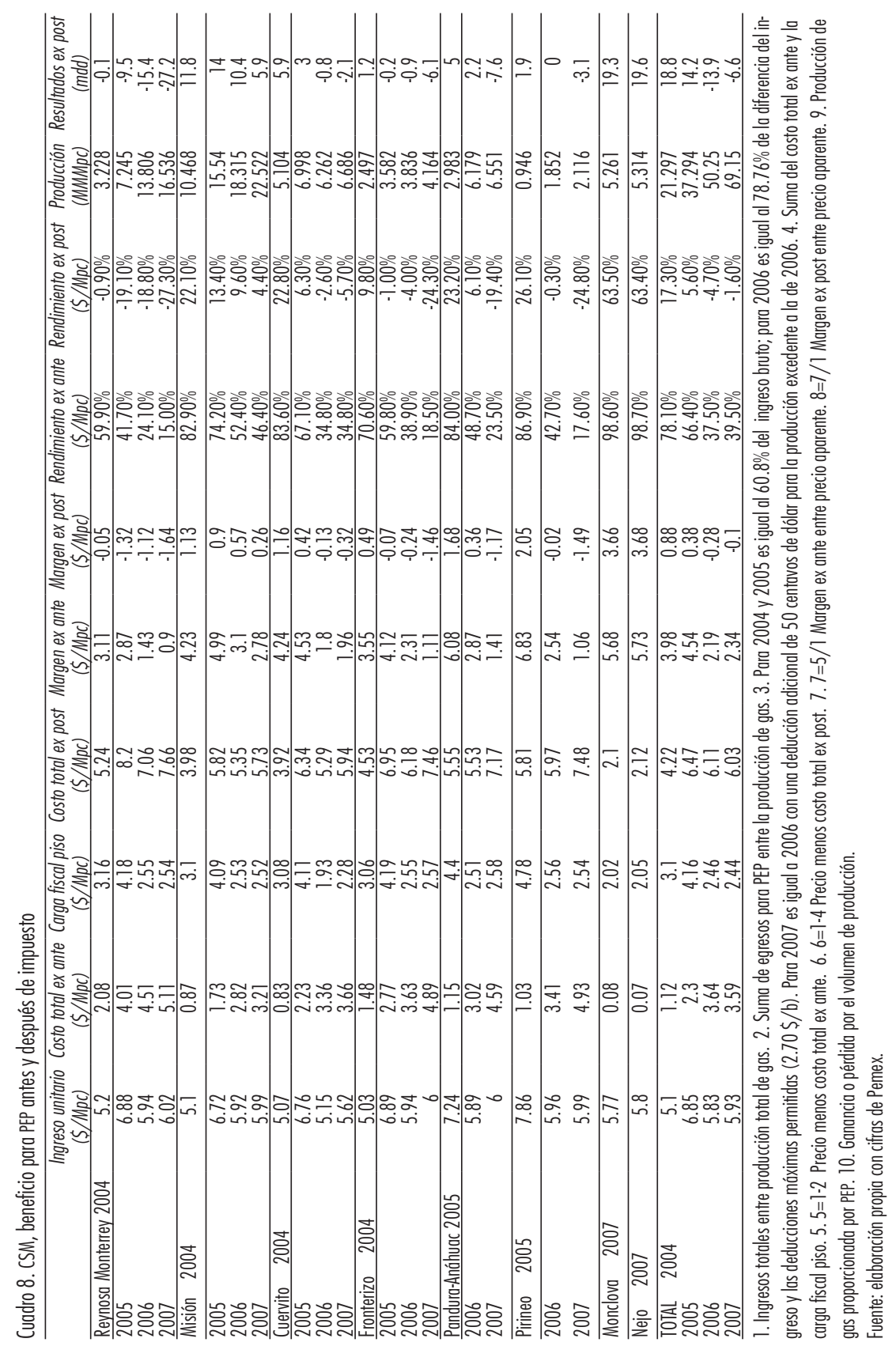


Víctor Rodríguez Padilla

\section{PROGRAMAS DE ENTRENAMIENTO, APOYO A LA COMUNIDAD Y CONTENIDO NACIONAL}

Los CSM prevén programas anuales de entrenamiento en tecnología de punta a cargo del contratista. ${ }^{15}$ En los PAT aparecen de manera esporádica y su grado de cumplimiento es deficiente (cuadro 9). Algunas veces se reportan gastos en entrenamiento de personal no previstos; en otros casos no se indican los montos asociados. PEP desconoce si el monto de dichos programas está bien calculado y si la asignación para cada uno de los programas fue correcta; además, distan de ser entrenamiento en tecnología de punta. ${ }^{16}$

Los CSM prevén programas anuales de apoyo a la comunidad que se deben incluir en el PAT. ${ }^{17}$ En la práctica rara vez se ha cumplido esa disposición. En otras ocasiones se incumple lo programado (cuadro 10). Los programas se han enfocado a mejorar las condiciones de escuelas, centros de salud, unidades de deportivas, parques públicos entre otras instalaciones. ${ }^{18}$ Sin embargo, Pemex no desglosa el monto destinado a cada una de las acciones emprendidas y no se puede saber si hay sobrefacturación.

La cláusula contractual en materia de contenido nacional establece que el contratista deberá dar preferencia a profesionales, trabajadores y demás empleados que sean de nacionalidad mexicana, cuando éstos cuenten con las calificaciones y experiencia requeridas para las obras. PEP tiene la obligación de verificar que esa cláusula se cumpla, pero en la práctica desconoce el número de efectivos que laboran en cada área de trabajo, su nacionalidad y competencias.

Los CSM establecen que el contratista dará preferencia tanto a la adquisición de bienes de origen mexicano, como a la contratación de servicios prestados por empresas mexicanas, bien sean privadas o estatales, siempre y cuando tales bienes y servicios se

15 En el caso de Reynosa Monterrey el contrato establece que el valor del programa deberá ser como mínimo del $0.25 \%$ del presupuesto presentado en el Programa Anual de Trabajo (PAT) cuando se trate del personal del contratista, y de 500 dólares por sector incluido en el área de trabajo cuando se trate del personal de PEP. En el caso de Pandura-Anáhuac se establece un valor de 520 dólares por sector para cada uno de dichos programas. En los casos de Nejo y Monclova los programas anuales de entrenamiento dejaron de ser en tecnología de punta y se incluyeron otras materias, cuyo valor será de no menos de $0.25 \%$ del presupuesto incluido en el PAT para personal del contratista y $1 \%$ para el personal de PEP.

16 En los programas de entrenamiento en tecnología de punta se reportan cursos, conferencias o talleres básicos de computación; inglés; aplicaciones del Logicial Office; creatividad; administración de proyectos; análisis económico y de riesgos; negocios; reforma fiscal. También se incluyen la asistencia a congresos de contadores públicos, diplomados en finanzas y otros rubros.

17 Por ejemplo, en el contrato Reynosa Monterrey se establece un programa de un valor mínimo de 500 dólares por sector incluido en el área de trabajo; los fondos serán destinados a obras que beneficien a PEP y a la comunidad pero también al contratista. En el caso del Pandura-Anáhuac el valor del programa subió a 520 dólares y se incluyó que el programa debería destinarse, además de lo señalado a acciones que contribuyan al desarrollo humano y sustentable, que beneficien tanto al contratista como a PEP y a la comunidad. En el caso de los contratos Monclova y Nejo se establece un valor mínimo de $2.25 \%$ del presupuesto correspondiente al PAT aprobado para el Ańo Contractual.

18 Algunos ejemplos de lo que incluyen los programas son los siguientes: "suministro de mobiliario", consistente en 6 cestos grandes y 4 cestos chicos para basura; "suministro de mobiliario", que incluye un equipo de sonido; "mobiliario", consistente en 3 pintarrones; suministro e instalación de un botiquín con medicamentos; instalación de 2 abanicos y 1 botiquín con medicamentos. 
Contratos de servicios múltiples en Pemex: eficacia, eficiencia y rentabilidad

Cuadro 9. Programas de entrenamiento al personal del contratista y de PEP (Dólares)

\begin{tabular}{|c|c|c|c|c|c|c|}
\hline & \multicolumn{3}{|c|}{ Programa Anual de Trabajo } & \multicolumn{3}{|c|}{ Real } \\
\hline Dólares & Personal del contratista & Personal de PEP & Total & Personal del contratista & Personal de PEP & Total \\
\hline \multicolumn{7}{|c|}{ Reynosa Monterrey } \\
\hline 20041 & * & * & * & $\star$ & * & 161,155 \\
\hline 2005 & 286,000 & 650,921 & 936,921 & * & * & 838,700 \\
\hline 20061 & * & * & * & * & * & 506,047 \\
\hline 20071 & * & * & * & & & 92,898 \\
\hline \multicolumn{7}{|l|}{ Misión } \\
\hline 20041 & * & * & * & * & * & 0 \\
\hline 20051 & * & * & * & * & * & 40,312 \\
\hline 20061 & * & * & * & * & * & 198,371 \\
\hline 20071 & * & * & * & * & * & 262,700 \\
\hline \multicolumn{7}{|c|}{ Pandura-Anáhuac } \\
\hline 2005 & 260,000 & 260,000 & 520,000 & * & * & 0 \\
\hline 2006 & 360,000 & 420,000 & 780,000 & & & 464,381 \\
\hline 20071 & * & * & * & * & * & 355,349 \\
\hline \multicolumn{7}{|c|}{ Pirineo } \\
\hline 20051 & * & * & * & * & * & 0 \\
\hline 20061 & * & * & * & * & * & 127,329 \\
\hline 20071 & * & * & * & * & * & 229,624 \\
\hline \multicolumn{7}{|c|}{ Monclova } \\
\hline 20071 & * & * & * & * & * & 0 \\
\hline \multicolumn{7}{|l|}{ Nejo } \\
\hline 20071 & * & * & * & * & * & 53,288 \\
\hline
\end{tabular}

* = Pemex no dispone de esa información. 1. PAT incompleto

Fuente: elaboración propia con cifras de Pemex.

encuentren en igualdad de condiciones en cuanto a precio, calidad, tiempo de entrega y demás condiciones relevantes a la subcontratación. En la práctica, PEP desconoce el contenido nacional de los bienes y servicios adquiridos en cada uno de los bloques en el periodo 2003-2007.

\section{DESEMPEÑO CON RESPECTO A LOS OBJETIVOS INICIALES}

Hemos señalado que PEP adoptó los CSM con el objetivo central de elevar la producción para sustituir con gas nacional barato las costosas y crecientes importaciones. En ese terreno los resultados han sido poco favorables: la producción ha estado muy por debajo de las metas y el costo de producción ha superado el de importación. El esfuerzo exploratorio 
Víctor Rodríguez Padilla

Cuadro 10. Programa apoyo a la comunidad

(Dólares)

\begin{tabular}{|c|c|c|c|c|c|}
\hline & PAT & Real & & PAT & Real \\
\hline \multicolumn{3}{|c|}{ Reynosa Monterrey } & \multicolumn{3}{|c|}{ Pandura Anáhuac } \\
\hline 2004 & $1 /$ & 596,029 & 2005 & 260,000 & 67,931 \\
\hline 2005 & 650,000 & 644,458 & 2006 & 425,000 & 335,771 \\
\hline 2006 & 1 & 585,622 & 2007 & 1/ & 30,895 \\
\hline 2007 & 1 & 74,180 & & & \\
\hline \multicolumn{3}{|c|}{ Misión } & \multicolumn{3}{|c|}{ Pirineo } \\
\hline 2004 & 1 & 0 & 2005 & 1/ & ** \\
\hline 2005 & 1 & 311,942 & 2006 & 1/ & 487,102 \\
\hline 2006 & 396,341 & 267,297 & 2007 & 1/ & 219,598 \\
\hline 2007 & 281,308 & 291,742 & & & \\
\hline \multicolumn{3}{|c|}{ Cuervito } & \multicolumn{3}{|c|}{ Monclova } \\
\hline 2004 & 1/ & 37,479 & 2007 & $1 /$ & 21,838 \\
\hline 2005 & 1 & 37,180 & & & \\
\hline 2006 & 1 & 14,822 & & & \\
\hline 2007 & 1 & 59,797 & & & \\
\hline \multicolumn{3}{|c|}{ Fronterizo } & \multicolumn{3}{|c|}{ Nejo } \\
\hline 2004 & 1 & 37,479 & 2007 & 1/ & *夫 \\
\hline 2005 & 1 & 37,180 & & & \\
\hline 2006 & 1 & 26,415 & & & \\
\hline 2007 & 1 & 56,863 & & & \\
\hline
\end{tabular}

Notas. 1 PAT incompleto (no incluye el programa de apoyo o su monto).

** Se recalendarizó para 2008.

Fuente: elaboración propia con cifras de Pemex..

ha sido débil y las reservas extraídas no han sido repuestas. Entre los objetivos secundarios algunos se han cumplido satisfactoriamente, pero otros sólo de manera moderada o escasa. En algunos casos la información disponible no permite expresar una opinión sólidamente documentada del grado de cumplimiento de las metas (cuadro 11):

a) Propiciar un desempeño del contratista más consistente con el resultado final que requería PEP no se cumplió. El organismo público creyó proponer un contrato con incentivos suficientes para que los contratistas se empeñaran en maximizar la producción, pero no fue así. Los contratistas han estado más interesados en realizar el mayor volumen de obras y servicios, aunque resulten redundantes o innecesarios, porque así obtienen una ganancia más importante. Ese problema se agregó a la lista de argumentos que justificaron la reforma energética de 2008. 
Contratos de servicios múltiples en Pemex: eficacia, eficiencia y rentabilidad

Cuadro 11. Desempeño de los CSM 2004-2008

\begin{tabular}{|c|c|c|c|c|}
\hline & \multicolumn{4}{|c|}{ Grado de cumplimiento } \\
\hline & Alto & Medio & Bajo & Incierto \\
\hline Incrementar reservas & & & $x$ & \\
\hline Incrementar producción & & & $x$ & \\
\hline Aportar capacidades operativas, tecnológicas y financieras de particulares & & $x$ & & \\
\hline Propiciar un desempeño más consistente con el resultado final deseado por PEP & & & $x$ & \\
\hline Incluir un sistema de financiamiento aportado por el contratista & & $x$ & & \\
\hline Ampliar las fuentes de financiamiento de PEP & & $x$ & & \\
\hline Mejorar las condiciones de financiamiento de los proyectos de PEP & & & & $x$ \\
\hline Reembolso de investiones y gastos a partir de la venta de la producción. & & $x$ & & \\
\hline Imprimir celeridad a los proyectos de incremento de la producción & & & $x$ & \\
\hline Ampliar a la disponibilidad de recursos de inversión de PEP & & $x$ & & \\
\hline Ampliar la capacidad de ejecución & $x$ & & & \\
\hline Maximizar el valor económico para PEP & & & $x$ & \\
\hline Propiciar condiciones competitivas mediante participación amplia de licitantes & & $x$ & & \\
\hline Menores costos de transacción por la administración de contratos & $x$ & & & \\
\hline Menor costo de suministro de gas respecto al costo de importación & & & $x$ & \\
\hline Ahorros potenciales en los costos de producción & & & $x$ & \\
\hline Mayor demanda de insumos nacionales & & & & $x$ \\
\hline Reducción del impacto de la deuda pública & & $x$ & & \\
\hline Flujo de efectivo positivo para el Estado antes de impuestos & $x$ & & & \\
\hline Flujo de efectivo positivo para el Estado antes de impuestos & & & $x$ & \\
\hline Acceso a tecnologías de punta & & & $x$ & \\
\hline Optimizar el desarrollo y la explotación de los yacimientos & & $x$ & & \\
\hline
\end{tabular}

b) Imprimir celeridad a la ejecución de los proyectos para incrementar la producción de gas no asociado no se cumplió porque la mayoría de los contratistas han incumplido los programas operativos anuales y las metas de producción.

c) Aportar capacidades operativas, tecnológicas y financieras de particulares se cumplió parcialmente, pues tres bloques quedaron desiertos. ${ }^{19}$

d) Incluir un sistema de financiamiento aportado por el contratista, ampliar las fuentes de financiamiento de PEP y pagar al contratista con los ingresos obtenidos por la venta de la producción se cumplieron, excepto en Olmos.

e) Ampliar con economía, eficacia y eficiencia la disponibilidad de recursos de inversión de PEP se ha cumplido parcialmente; varios bloques quedaron desiertos y no se concretaron las inversiones esperadas. Por lo que toca a las condiciones de

19 Se trata de los bloques Ricos, Corindón Pandura y Euro. 
Víctor Rodríguez Padilla

financiamiento de los proyectos de PEP no se tiene información suficiente para concluir que éstas mejoraron.

f) Reducir el impacto de la deuda pública se cumplió pues los CSM se estructuraron como Proyectos de Infraestructura Diferidos en el Registro del Gasto (Pidiregas). Obtener un flujo de efectivo positivo para el Estado se cumplió, pero PEP registra pérdidas después de impuestos.

g) Ampliar con economía, eficacia y eficiencia las capacidades de ejecución de PEP no se cumplió, en primer lugar porque capacidad de ejecución no es sinónimo de capacidad de contratar; aunque las firmas privadas actúen por cuenta y obra del organismo, las capacidades operativas, tecnológicas y financieras son estrictamente de los contratistas. En segundo lugar porque algunas licitaciones quedaron desiertas.

h) Maximizar el valor económico para PEP no se cumplió, pues los gastos crecieron más rápido que los ingresos al no cumplirse las metas de producción.

i) Propiciar condiciones competitivas para el Estado a través de una participación amplia de licitantes, no se cumplió porque sólo se presentaron uno o dos licitantes por bloque y algunas licitaciones quedaron desiertas.

j) Obtener menores costos de transacción por la administración de contratos se cumplió, pues cuesta menos administrar un contrato que muchos.

k) Obtener menores costos de suministro del gas respecto al costo de importación. Se logró parcialmente, pues tomando en cuenta el régimen fiscal, PEP pierde por cada pie cúbico entregado por los CSM. Obtener ahorros potenciales en los costos de producción no se cumplió, pues dichos costos aumentaron. Obsérvese que con o sin deriva de costos los ahorros potenciales han sido absorbidos por los contratistas porque el rembolso de inversiones y gastos se realiza con base en los precios unitarios de las obras y servicios. ${ }^{20}$

l) No contamos con información suficiente para saber si aumentó la creación de empleos y la demanda de insumos nacionales, por arriba de lo que hubiera conseguido PEP con sus propios medios y no mediante CSM. No hay evidencias de que los contratistas adquieran más insumos nacionales; la lógica indica lo contrario pues las firmas extranjeras tienden a contratar a las empresas de su propia nacionalidad.

m) Aumentar los conocimientos y solvencia técnica de PEP no se ha cumplido porque los programas de formación del personal no han sido en tecnologías de punta. No se cuenta con información suficiente para saber si los contratistas están utilizando tecnología de punta en las operaciones cotidianas.

n) Optimizar el desarrollo y la explotación de los yacimientos no se cumplió porque la función objetivo de los contratistas han sido maximizar la cantidad de obras y servicios.

20 El contrato está diseñado para que las ganancias de eficiencia y productividad se las quede el contratista; forma parte del conjunto de incentivos para interesar a los inversionistas. 
Como se observa, de la larga lista de objetivos que se propuso PEP pocos se han logrado y no hay evidencias de que en el futuro será diferente debido al esquema de incentivos contenido en el contrato.

\section{CONCLUSIÓN}

Pemex puso en marcha los CSM con el objetivo de ampliar con economía, eficacia y eficiencia las fuentes de financiamiento y las capacidades de ejecución de PEP en materia de gas no asociado, al mismo tiempo, para maximizar el valor económico para el organismo, obtener un menor costo de suministro de gas respecto al costo de importación y ahorros potenciales en el costo de producción. Supuso que la contratación de servicios de manera integral daría mejores resultados que el método tradicional de contratar de manera aislada cada servicio. Los resultados han sido desiguales.

El desempeño económico de los CSM ha sido poco favorable en términos de eficacia, eficiencia y rentabilidad para PEP. El organismo supuso que el gas producido mediante CSM sería más barato que el producido mediante otras modalidades contractuales e incluso del gas importado del sur de Texas. Esa hipótesis no se cumplió. Tampoco se cumplió la tesis de un rápido y vigoroso aumento de la producción gracias a esos contratos; la meta original de alcanzar mil millones de pies cúbicos a partir de 2007 se ha cumplido en menos de $20 \%$. En cinco de los nueve contratos vigentes PEP pierde dinero después de impuestos. En el plano de los objetivos secundarios se observan claro oscuros: algunos se han cumplido a plenitud pero otros sólo de manera parcial o escasa.

Más allá del diagnóstico y los pronósticos que soportaron la decisión de poner en marcha los CSM y de los pálidos resultados obtenidos, es claro que PEP ha ganado conocimientos y experiencia en el diseño, aplicación y supervisión de contratos de exploración y producción de uso corriente en la industria petrolera internacional, situación inédita desde hace varias décadas que prepara el camino para nuevas modalidades contractuales y un modelo de explotación petrolera distinto al monopolio público. 
Víctor Rodríguez Padilla

\section{BIBLIOGRAFÍA}

Auditoría Superior de la Federación, Informe del Resultado de la Revisión y Fiscalización Superior de la Cuenta Pública 2007, Tomo viI, Volumen 3, Sector Energía, México DF, 623 p.

Bourgeois, B. y V. Rodríguez Padilla, "Les nouveaux schémas d'association entre pays producteurs et pays consommateurs de pétrole", Le Pétrole et le Gas Arabes, XXIV (547), $1^{\circ}$ enero, 1992, pp. 36-42.

Muñoz Leos, Raúl, “The Pemex Gas Strategy”, Official International Conference Mexico's Gas Sector, Mexico City, 20-21 June, 2002.

Rodríguez Padilla V., "Petroleum and Nationalism", en Encyclopedia of Energy, New York, Academic Press, 2004.

Ramírez Corzo, Luis, "Public Works Contracting Process on the basis of Unit Prices, Official International Conference Mexico’s Gas Sector, Mexico City, 20-21 June, 2002.

Petróleos Mexicanos, Base Institucional de Datos. 